\title{
Research on Communication Signal Filtering Method Based on Generalized S-Transform
}

\author{
Ruowu Wu' ${ }^{1}$, Yongzhi Wang ${ }^{2}$, Hui Han ${ }^{1}$, Xiang Chen ${ }^{1}$, Yun Lin ${ }^{2, *}$ \\ 1. State Key Laboratory of Complex Electromagnetic Environment Effects on Electronics and \\ Information System (CEMEE), Luoyang, Henan, 471003, China \\ 2. College of Information and Communication Engineering, Harbin Engineering University, \\ Harbin, China \\ Corresponding Author: Yun Lin, linyun_phd@hrbeu.edu.cn
}

\begin{abstract}
In multimedia signal, because of the existence of Gaussian white noise in the environment, the speech signal is very vulnerable to pollution. In order to filter noise and extract useful components from signals, a speech signal filtering method based on generalized S transform and image processing is proposed in this paper. The method is compared with other two kinds of filtering methods. The simulation results show that, in the range of signal to noise ratio (SNR) $10 \mathrm{~dB} \sim 10 \mathrm{~dB}$, the filtering method can effectively improve the SNR more than $5 \mathrm{~dB}$, and its performance is the best.
\end{abstract}

Keywords: multimedia signal processing; generalized $S$ transform; timefrequency filtering

\section{Introduction}

With the development of science and technology, especially the communication technology, people's ability to recognize and apply signals is improving. Various kinds of signal processing methods emerge in an endless stream. Signal filtering technology has made great progress. In multimedia signals, speech signals are easily disturbed by noise, so the main purpose of filtering is to eliminate the influence of background noise on useful signals. As a typical non-stationary signal, there are many ways to filter speech signals, such as linear canonical transform filtering, wavelet filtering, Wiener filtering, $\mathrm{S}$ transform filtering and generalized $\mathrm{S}$ transform filtering. In recent years, many scholars have studied and discussed this method.

S transform (ST) [1] and the generalized S transform (GST) are good timefrequency analysis methods that satisfy linear superposition without the interference of cross terms, and the time-frequency resolution can change with the frequency. Now ST and GST are widely used in speech signal processing, medical image processing, and seismic signal processing and so on. In 2013, Yin et al. [2] proposed a singular value decomposition (SVD) filtering method based on GST when studying the filtering problem of cardiac magnetic signal. In this method, the generalized $\mathrm{S}$ transform is used 
to adjust the time-frequency resolution, and the SVD method is used to identify effective cardiac magnetic signal area, so as to achieve adaptive time-frequency filtering. In 2015, document [3] proposed a Gaussian neighborhood filtering method based on GST, and discussed the detailed design process of filtering domain corresponded to different filter models. Li Hongguang et al. [4] selected the appropriate threshold ratio coefficient to filter the seismic signal by analyzing the GST timefrequency characteristics of the background noise's Green function. Document [5] proposed a time-frequency filtering method based on GST. By calculating the distance from each point to the instantaneous frequency in the time-frequency plane, the normalized distance set was obtained, and based on which, a filter was built for the analysis and processing of LFM signal.

In this paper, the LFM signal is used as an example to apply the generalized $S$ transform to the filter, and a filtering method based on image processing is proposed. By comparing the method with the filter methods based on SVD-fuzzy C mean clustering algorithm and the CFAR detection method respectively, it is proved that the algorithm proposed in this paper is most effective. In addition, by comparing the filtering effect of GST, ST and STFT, it is proved that GST has the best filtering effect.

\section{Algorithm introduction}

\section{1. $S$ transform and generalized $S$ transform}

$\mathrm{S}$ transform is a linear time frequency analysis method proposed by $\mathrm{R}$. G. Stockwell et al. in 1996. The $\mathrm{S}$ transform formula of the signal $x(t) \in L^{2}(R)$ is as follows:

$$
S(t, f)=\int_{-\infty}^{+\infty} x(\tau) w(\tau-t) e^{-j 2 \pi f \tau} d \tau
$$

In which the window function is $w(t)=\frac{1}{\sigma \sqrt{2 \pi}} e^{-\frac{t^{2}}{2 \sigma^{2}}} \cdot \sigma$ is the scale factor function. Window function satisfies $\int_{-\infty}^{+\infty} w(\tau-t) d \tau=1$. In standard $\mathrm{S}$ transform, scale factor function $\sigma(f)=\frac{1}{|f|}$. The inverse $\mathrm{S}$ transform (IST) can be realized by the inverse Fourier transform.

$$
x(t)=\int_{-\infty}^{+\infty}\left[\int_{-\infty}^{+\infty} S(\tau, f) d \tau\right] e^{j 2 \pi f t} d f
$$

GST is a time frequency analysis method based on the idea of ST. The generalized $S$ transformation proposed by Chen Xuehua in $2005^{[6]}$ introduced two parameters $\lambda$ and $p$. Different from ST, the scale factor function is changed to $\sigma(f)=\frac{1}{\lambda|f|^{p}}$. Window function $w(t)=\frac{\lambda|f|^{p}}{\sqrt{2 \pi}} e^{-\frac{t^{2} \lambda^{2} f^{2 p}}{2}}$ 
The GST of signal $x(t)$ is as follows:

$$
\operatorname{GST}(t, f)=\int_{-\infty}^{+\infty} x(\tau) \frac{\lambda|f|^{p}}{\sqrt{2 \pi}} e^{-\frac{(\tau-t)^{2} \lambda^{2}|f|^{2 p}}{2}} e^{-j 2 \pi f \tau} d \tau
$$

In which $\lambda>0, p>0$. Similar to ST, the inverse transformation of GST is also lossless and reversible. The formula for the inverse transformation of GST is:

$$
x(t)=\int_{-\infty}^{+\infty}\left[\int_{-\infty}^{+\infty} G S T(\tau, f) d \tau\right] e^{j 2 \pi f t} d f
$$

\subsection{Filtering method based on image processing}

Time frequency diagram can be regarded as a grayscale image. Every element in the time-frequency matrix is a pixel point. The size of the element value is reflected in the size of the pixel gray level in the image, so we can use the image processing method to analyze the time-frequency map. The process of image processing is shown in Figure.1.

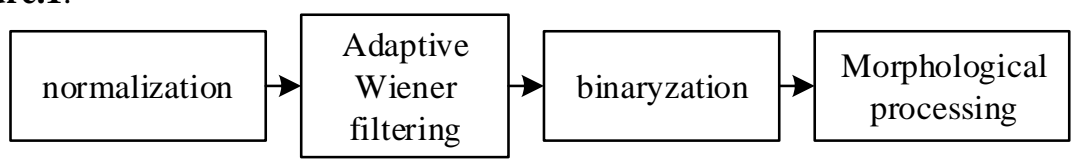

Fig. 1. Basic steps of time frequency image processing

Wiener filter is a linear smoothing filter, which adaptively adjusts the output of the filter according to the region variance of the image. When the local variance is large, the smoothing effect of the filter is weak, otherwise, it is stronger. The Wiener filter first estimates the local mean and local variance of the pixels:

$$
\begin{gathered}
\mu=\frac{1}{M N} \sum_{n_{1}, n_{2} \in \eta} a\left(n_{1}, n_{2}\right) \\
\sigma^{2}=\frac{1}{M N} \sum_{n_{1}, n_{2} \in \eta} a^{2}\left(n_{1}, n_{2}\right)-\mu^{2}
\end{gathered}
$$

Where, $\eta$ is the $M \times N$ neighborhood of each pixel in the image. Then each pixel is estimated with a Wiener adaptive filter for its gray value:

$$
b\left(n_{1}, n_{2}\right)=\mu+\frac{\sigma^{2}-v^{2}}{\sigma^{2}}\left(a\left(n_{1}, n_{2}\right)-\mu\right)
$$

Where, $v^{2}$ is the variance of the noise in the image, which can be replaced by the mean of all local estimated variance. Due to the influence of noise, some Gauss white noise still exists in the form of small connected domain after binaryzation. In order to filter out the noise of this part, we remove the small objects from the object. The main steps are:

a. Determines the connected object;

b. Calculates the area of each connected object;

c. removes objects less than $\mathrm{P}$. Here $\mathrm{P}$ is a threshold for the removal of the object area according to the needs.

Small and meaningless objects can be eliminated by the use of corrosion operations. The concept of corrosion in the general sense is defined as ${ }^{[7]}$ : 


$$
E(x, y)=(I \otimes T)(x, y)=\underset{i, j=0}{A N}[I(x+i x) \& T(i, j)]
$$

Which is to say, the two-dimensional image $E$ produced by corrosion of $T$ by $I$ is a set of points $(x, y)$ that meet the following condition: If the origin of $I$ translates to the point $(x, y)$, then $I$ will be completely included in the $T$.

Contrary to the corrosion operation, the expansion operation is the process of expanding the boundary of the image to the outside. Using this operation, the space in the object can be filled. The concept of expansion in general meaning is defined as:

$$
D(x, y)=(I \otimes T)(x, y)=\stackrel{m}{O R}[I(x+i, y+j) \& T(i, j)]
$$

That is to say, the binary image $D$ generated by the expansion of $T$ by $I$ is a set of points $(x, y)$ that satisfy the following condition: If the origin of $I$ translates to the point $(x, y)$, then the intersection of $I$ and $T$ is not empty.

Through the above image processing steps, the original time frequency image has now become a binary image with more regular edges and less noise. In order to reconstruct the original signal, we use the binary image as the filter to deal with the original image. The binary image matrix after processing is assumed to be $H(t, f)$, in which the value of $f$ is 1 or 0 . Then, the time frequency matrix $P_{x}{ }^{\prime}(t, f)$ is obtained by multiplying $H(t, f)$ with the original time frequency matrix $P_{x}(t, f)$, thus:

$$
P_{x}{ }^{\prime}(t, f)=P_{x}(t, f) \square H(t, f)
$$

In order to reconstruct the original time series, based on the reversibility of the linear time frequency transformation, the inverse GST of $P_{x}{ }^{\prime}(t, f)$ is carried out, and the original time series $x^{\prime}(t)$ is reconstructed. And for linear time-frequency analysis methods GST, ST and STFT, the signal to noise ratio of the reconstructed signal is used to evaluate the filtering effect respectively.

\section{Simulation results}

Set the starting frequency of the LFM signal $f_{0}=1 \mathrm{kHz}$ and the termination frequency $f_{1}=2 \mathrm{kHz}$. The number of sampling points for time is 256 . The sampling frequency is $20 \mathrm{kHz}$, and the signal to noise ratio is $-10 \mathrm{~dB} \sim 10 \mathrm{~dB}$. The parameters of the window function of GST are obtained by the optimization of genetic algorithm, which are $\lambda=17.7929$ and $p=0.0001$ respectively. In order to ensure the results have statistical significance, experiment was achieved across 20 Monte-Carlo in each SNR and averaged. The simulation results are shown in Figure 2.

It can be seen from the graph that with the increase of SNR, the SNR of reconstructed signal is increasing. When the original signal-to-noise ratio is $-10 \mathrm{~dB}$, the three time frequency analysis methods can achieve 5dB SNR increase. When the original SNR is $10 \mathrm{~dB}$, the reconstructed SNR of STFT reaches 20dB, which improves 
$10 \mathrm{~dB}$, while GST and ST can also reach $15 \mathrm{~dB}$, and SNR can be increased by more than $5 \mathrm{~dB}$. Compared with GST and ST at the same SNR, the filtering effect of GST is better than that of ST. When the original SNR is lower than OdB, the filtering effect of GST is better than that of STFT. When the original SNR is $0 \mathrm{~dB} \sim 3 \mathrm{~dB}$, the filtering effect of GST and STFT are similar. When the original SNR is higher than 3dB, STFT's filtering effect is better than GST, and STFT is more suitable for filtering environment with high SNR.

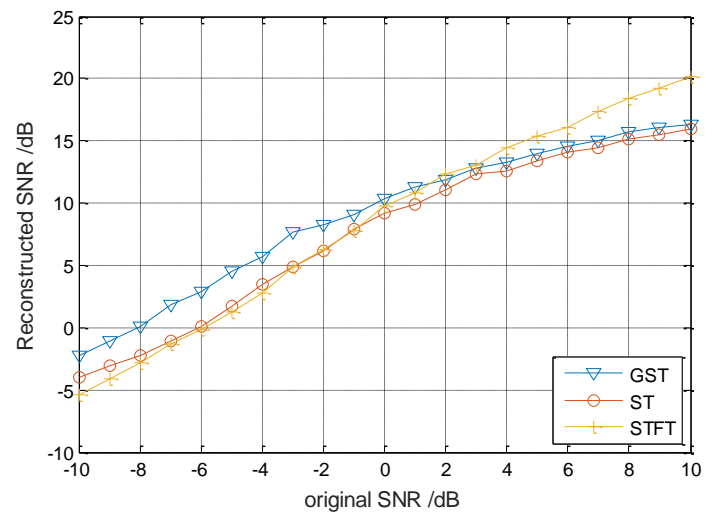

Fig. 2. Comparison of GST image processing and filtering methods for LFM signal

Through the research and Simulation of the above three time-frequency filtering methods, it is obvious that the GST algorithm is more suitable for low SNR environment than ST and STFT, and has great application value in engineering. In the three filtering methods, although the first and third lifting ranges are very close, the image processing filtering method has been able to increase the signal-to-noise ratio to $10 \mathrm{~dB}$ at $0 \mathrm{~dB}$, which is obviously more effective.

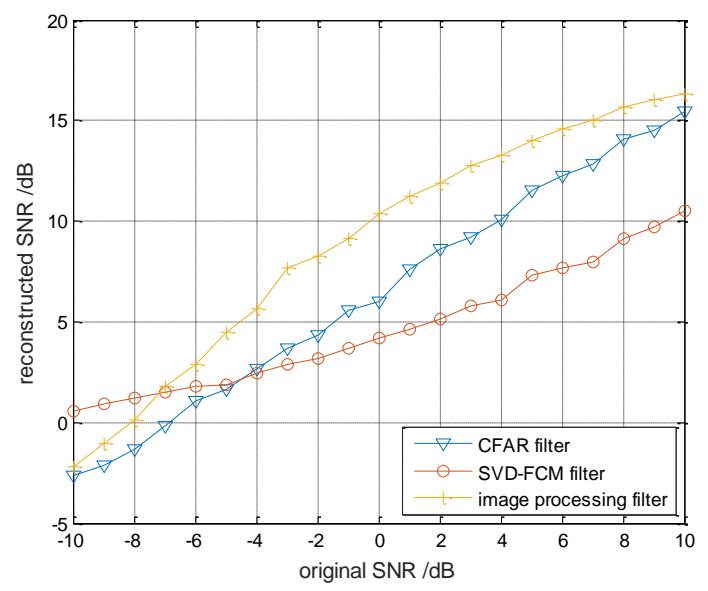

Fig. 3. Comparison of filtering effect of GST algorithm 
For the GST algorithm, Figure 3. gives the comparison of the filtering effect of the three filtering methods. It can be seen from the graph that when the SNR is less than $7 \mathrm{~dB}$, the filtering effect of SVD-FCM is the best, especially when the SNR is $-10 \mathrm{~dB}$, SVD-FCM's filtering method can increase the SNR to more than OdB. When the SNR is greater than $-7 \mathrm{~dB}$, the filtering effect of image processing is obviously better than the other two filtering methods. Considering with the increase of the SNR, the filtering effect of SVD-FCM filter gradually weakened, so that in the 10dB when the SNR improvement is not obvious, it can be concluded that this filtering method has some limitations. In the $-10 \mathrm{~dB}$, although the image processing filtering method of the filter effect is worse than SVD-FCM, the SNR is still improved by $7 \mathrm{~dB}$. From the simulation results in the range of $\mathrm{SNR}-10 \mathrm{~dB} \sim 10 \mathrm{~dB}$, the filtering effect based on image processing method is better than that based on constant false alarm rate detection and SVD-FCM filtering.

\section{Conclusion}

This paper mainly introduces a GST filtering method based on image processing. The method is compared with the constant false alarm rate filtering and the singular value decomposition based filtering method. The filtering effect is compared with GST and ST and STFT respectively, and the signal to noise ratio of reconstructed signal is used to evaluate the filtering effect. The results showed that the performance of GST was the best in any time frequency analysis. In these three filtering methods, the filtering effect based on image processing is better than the other two.

\section{Acknowledgments.}

This paper is supported by the National Natural Science Foundation of China (61301095) and the State Key Lab of CEMEE Foundation (CEMEE2018K0104A)

\section{References}

[1] Stockwell, R. G., Mansinha, L., Lowe, R. P: Localization of the complex spectrum: the $\mathrm{S}$ transform. IEEE Press. (1996)

[2] Yin Boqiang, He Yigang, Wu Xianming: Singular value decomposition filtering method in generalized $\mathrm{S}$ transform domain of magnetic signals. Journal of Physics, Vol. 62, Nu.14, pp. 504-511 (2013)

[3] Jin Zhi, Yin Boqiang: Gauss domain time-frequency filtering method based on generalized S transform. Journal of electronic measurement and instrument, Vol.29, Nu.1, pp. 124-131 (2015)

[4] Li Hongguang, Sun Guang, Xing Yilan: Application of generalized S transform 
in background noise data processing. Journal of Seismology Engineering, Vol.37, Nu.3, pp. 867-872 (2015)

[5] Wang, D., Wang, J., Liu, Y., Xu, Z: An adaptive time-frequency filtering algorithm for multi-component LFM signals based on generalized S-transform. International Conference on Automation and Computing. IEEE.Vol.129, pp.1-6. (2015).

[6] Chen Xuehua, He Zhenhua, Huang Deji: Signal extraction and noise suppression based on generalized S transform. Journal of Chengdu University of Technology (NATURAL SCIENCE EDITION), Vol.33, Nu.4, pp.331-335. (2006)

[7] Ma Chengying:. The method and application of time frequency attribute extraction. Doctoral dissertation of Ocean University of China. (2012) 